\title{
DESENVOLVIMENTO DE QUEIJO TIPO COTTAGE SEM LACTOSE COM ADIÇÃO DE FIBRAS E REDUÇÃO DE SÓDIO E GORDURA
}

\section{Development of cottage cheese lactose free with fiber addition and reduction of sodium and fat}

\author{
Gabrieli Nicoletti $^{1^{*}}$, Kátia Joana Verdi ${ }^{l}$, Creciana Maria Endres ${ }^{l}$
}

\begin{abstract}
RESUMO
O objetivo deste trabalho foi desenvolver, caracterizar e determinar a vida útil de queijo tipo cottage sem lactose, com adição de fibras e redução de sódio e gordura. Foram realizados testes experimentais para determinar a formulação final do queijo, a qual foi avaliada em diferentes tempos de armazenamento, de acordo com ensaios microbiológicos preconizados pela legislação vigente e paramêtros físico-químicos. O produto desenvolvido atendeu aos padrões físico-químicos se comparado ao queijo controle, apresentando $41 \%$ e 30\% de redução de sódio e gordura, respectivamente e acréscimo de aproximadamente 5,31\% de inulina, sem alterar as características sensoriais. O queijo apresentou estabilidade durante os 42 dias avaliados, não sofrendo nenhuma alteração físico-química (acidez) e microbiológica significativa que comprometesse a segurança e qualidade do produto. A adição de fibras e a redução de sódio também não influenciaram negativamente na sensorialidade do produto, sendo uma alternativa de produtos isento de lactose e com propriedades funcionais para o mercado de lácteos.
\end{abstract}

Palavras-chave: produto lácteo; intolerância à lactose; alimentos funcionais.

\begin{abstract}
The objective of this work was to develop, characterize and determine the shelf life of lactose-free cottage cheese with fiber addition and sodium and fat reduction. Experimental tests were performed to determine the final cheese formulation,

1 Instituto SENAI/SC de Tecnologia de Alimentos e Bebidas, Rua Frei Bruno, 201, bairro Parque das Palmeiras, 89803-785, Chapecó, SC, Brasil. E-mail: gabrieli.nicoletti@sc.senai.br

* Autor para correspondência.
\end{abstract}

Recebido / Received: 12/02/2016

Aprovado / Approved: 07/06/2017 
which was evaluated at different storage times according to microbiological tests recommended by the current legislation and physicochemical parameters. The product developed met the physical-chemical standards when compared to the control cheese, presenting 41 and $30 \%$ reduction of sodium and fat, respectively, and an increase of approximately $5,31 \%$ of inulin, without altering the sensorial characteristics. The cheese presented stability during the 42 days evaluated, not suffering any physicalchemical alterations (acidity) and microbiological significant that compromised the safety and quality of the product. The addition of fiber and sodium reduction also did not negatively influence the sensoriality of the product, being an alternative of products lactose free and with functional properties for the dairy market.

Keywords: dairy product; lactose intolerance; functional food.

\section{INTRODUÇÃO}

O cottage é um tipo de queijo de massa fresca, de sabor levemente ácido e salgado, produzido pela coagulação ácida do leite desnatado pasteurizado. A característica principal deste queijo é sua textura granular, com partículas de massa coalhada de tamanho relativamente uniforme, que podem ou não estar imersas em um líquido cremoso (dressing). Contém cerca de $80 \%$ de umidade, com baixo teor de lipídio (4\%) e apresenta-se com dressing em duas fases separáveis (VIANNA et al., 2002).

Existem diversas variedades de queijo cottage, com diferenças na consistência, teores de gordura e de umidade. No Brasil, é comercializado na forma de grãos secos, grãos misturados ao dressing e, também, na forma pastosa (RODRIGUES, 1999). A legislação brasileira, até o momento, não possui padrões de identidade e qualidade específicos para o queijo cottage, o que dificulta comparações entre diferentes marcas comerciais, com base na legislação (PARODIA, 2010).

$\mathrm{O}$ interessante desta variedade em sua forma original é que, apesar de seu baixo conteúdo de gordura, apresenta características sensoriais muito apreciáveis, principalmente seu aroma e gosto, devido à sua condição de produto fermentado, uma vez que os microrganismos utilizados durante o processo deixam as características sensoriais muito atrativas (BRITO et al., 2006).

Devido à alta incidência de doenças crônicas nos últimos anos, a demanda por alimentos saudáveis tem estimulado inovações e desenvolvimento de novos produtos por todo o mundo. Ações que visam ao melhoramento nutricional dos alimentos processados têm apresentado uma maior disseminação, principalmente em relação à redução de gorduras, açúcares e sódio (OBANDO et al., 2010).

Os alimentos prebióticos fazem parte do grupo dos alimentos funcionais, pois além de suas qualidades nutricionais, afetam beneficamente uma ou mais funções relevantes do organismo do consumidor (RIBEIRO et al., 2009). Esta classe é definida como oligossacarídeos que não são digeríveis no intestino delgado e atingem o intestino grosso onde atua estimulando seletivamente o crescimento de bactérias bífidas residentes no trato gastro digestório, alterando a microbiota em favor de uma composição mais saudável. Os oligossacarídeos, como os fruto-oligossacarídeos e a inulina atendem as condições de prebióticos (MANNING et al., 2004).

Além disso, muitos estudos mostram que uma percentagem significativa da população mundial sofre com transtornos gastrointestinais quando consome produtos lácteos. 
Tais sintomas são provenientes da deficiência ou ausência da enzima lactase, responsável pela quebra da molécula de lactose, o principal açúcar do leite, sendo este transtorno chamado de intolerância à lactose. Esta problemática priva o intolerante à lactose da ingestão de produtos lácteos que são uma excelente fonte de cálcio e proteínas, como também de outros minerais e vitaminas (ANTUNES; PACHECO, 2009).

O presente trabalho teve por objetivo desenvolver um queijo tipo cottage com adição de inulina, isento de lactose e com teor reduzido de sódio e gordura e caracterizá-lo em relação aos parâmetros físico-químicos, sensoriais e microbiológicos durante o período de 42 dias de estocagem refrigerada $\left(5 \pm 2{ }^{\circ} \mathrm{C}\right)$.

\section{MATERIAL E MÉTODOS}

\section{Elaboração do queijo tipo cottage}

O estudo foi desenvolvimento no laboratório de processamento de leites e derivados do Instituto SENAI/SC de Alimentos e Bebidas, localizado na cidade de Chapecó/SC. Foram avaliados dois tratamentos, o queijo controle (sem adição de inulina, sem redução de gordura, lactose e sódio), considerada uma composição da literatura, fabricado da mesma maneira que o produto desenvolvido nesta pesquisa. O queijo tipo cottage (com redução de sódio, gordura, lactose e adição de inulina) foi fabricado de acordo com a formulação determinada na Tabela 1. Os ensaios foram realizados em triplicata.

A formulação do queijo tipo cottage foi desenvolvida com embasamento teórico através de pesquisas e artigos já publicados relacionados à adição de fibras em produtos lácteos. Foram realizados testes experimentais, respeitando o Regulamento Técnico de Identidade e Qualidade de Queijos, apresentado pela Portaria $n^{\circ} 146$ de 07 de março de 1996 (BRASIL, 1996), até que se encontrasse um produto característico.

A Tabela 1 apresenta a formulação definitiva do produto final. Salienta-se que para produzir $1 \mathrm{~kg}$ de produto final, foram utilizados 3,55 L de leite desnatado. Os percentuais adicionados estão estabelecidos em intervalos, dessa forma, mantém em sigilo a formulação desenvolvida em parceria com a empresa.

O queijo tipo cottage foi elaborado a partir de leite desnatado padronizado a $0,1 \%$ de gordura $(\mathrm{m} / \mathrm{v})$, após o mesmo foi hidrolisado com a enzima lactase (Maxilact ${ }^{\circledR}$ LGX5000, DSM, Holanda), para que ocorresse

Tabela 1 - Formulação para queijo tipo cottage com adição de fibra e redução de sódio

\begin{tabular}{lcc}
\hline \multicolumn{1}{c}{ Ingredientes } & Unidade de medida & Percentual $(\%)$ \\
\hline Dressing & $\mathrm{L}$ & 7 a 9 \\
- creme (16\% de gordura) & $\mathrm{L}$ & $95-98$ \\
- sal de sódio & $\mathrm{g}$ & $1-1,9$ \\
- sal de potássio & $\mathrm{g}$ & $1,15-1,60$ \\
Sorbato de potássio & $\mathrm{g}$ & $0,15-0,85$ \\
Inulina & $\mathrm{g}$ & $3-5,5$ \\
Massa básica & $\mathrm{g}$ & $64-69$ \\
Dressing + inulina + sorbato & $\mathrm{g}$ & $36-41$
\end{tabular}

* Formulação estabelecida para $1000 \mathrm{~g}$ de produto. 
a hidrólise da lactose. O processo de hidrólise foi realizado na temperatura de $37^{\circ} \mathrm{C}$ por três horas na proporção de $0,1 \%$ de enzima em relação ao volume de leite. A conversão dos açúcares foi avaliada através de ensaio laboratorial, adaptado por Harvey (1988).

Posterior a este processo, o leite foi submetido à pasteurização e inativação enzimática, essa foi realizada na temperatura de $60{ }^{\circ} \mathrm{C}$ a $65^{\circ} \mathrm{C}$ por 30 minutos. Após a pasteurização, procederam-se a fermentação, corte da massa, cozimento, lavagens, adição do dressing (leite, creme de leite e sal de sódio e potássio), envase e estocagem.

Para realização do processo de fermentação foram utilizadas as culturas liofilizadas para inoculação direta (DVS) composta por Lactococcus lactis spp. lactis, Lactococcus lactis spp. cremoris, Lactococcus lactis spp. lactis biovar. diacetylactis e Leuconostoc spp. (DELVO ${ }^{\circledR}$ - TECDX33 ADSL, DSM, Holanda), na proporção de $1 \%$ a $3 \%$ e aguardou-se 30 minutos para hidratação da cultura. Após esta etapa, adicionou-se solução de cloreto de cálcio a $50 \%$ e o coagulante, conforme recomendado pelo fabricante, na proporção de $0,04 \%$ e $0,06 \%$ respectivamente. Aguardou-se aproximadamente 120 minutos até a coagulação completa. Ao final da coagulação, o pH alcançado foi de $\mathrm{pH} 4,6-4,7$, acidez de $65^{\circ} \mathrm{D}$ $-70^{\circ} \mathrm{D}$, além da massa se apresentar homogênea, lisa e firme.

O corte da coalhada foi realizado com a utilização de liras horizontais e verticais para promover cortes em cubos pequenos (1,0 a 1,5 cm de aresta) que foi deixada em repouso durante 15 a 20 minutos, para que o grão de coalhada adquirisse a consistência. Ao fim deste tempo, iniciou-se o processo de aquecimento, com agitação da coalhada, de forma lenta para impedir que os grãos se aglomerassem. Para a realização do aquecimento foi retirada uma quantidade de soro que foi substituída por igual quantidade de água a $45{ }^{\circ} \mathrm{C}(5 \%$ em relação ao volume de leite trabalhado). A água foi adicionada de forma cautelosa para que os grãos, ainda frágeis, não fossem danificados e em seguida agitou-se durante 20-30 min. Então, iniciou o aquecimento da coalhada e concomitante a sua agitação, essa etapa foi feita de forma que, ao fim de uma hora ou uma hora e meia, a temperatura da coalhada atingisse $44^{\circ} \mathrm{Ca}$ $48{ }^{\circ} \mathrm{C}$ com aumento de aproximadamente $1{ }^{\circ} \mathrm{C}$ a cada 3-5 minutos. Salienta-se que o aquecimento deve ser indireto e gradativo de maneira a não "queimar" os grãos (formação de película envolta do grão). Após atingir a consistência ideal do grão, o qual deve ser firme, retirou-se todo o soro e iniciou o processo de lavagem da massa, adicionando-se uma boa quantidade de água pasteurizada com temperatura em torno de $40{ }^{\circ} \mathrm{C}$. Escorreu-se toda a massa e lavou com água pasteurizada com temperatura em torno de $30{ }^{\circ} \mathrm{C}$. A última lavagem foi feita com água pasteurizada e gelada (máxima de $5^{\circ} \mathrm{C}$ ). Nesta última etapa os grãos ficaram soltos e sem presença de soro. Por conseguinte, pesou-se a massa obtida e armazenou-se a temperatura máxima de $5{ }^{\circ} \mathrm{C}$, até misturar o dressing.

$\mathrm{Na}$ elaboração do dressing, inicialmente realizou-se a hidrólise do creme com $16 \%$ de teor de gordura, com utilização de $0,1 \%$ de enzima lactase na temperatura de $37{ }^{\circ} \mathrm{C}$ por um período de 2 a 3 horas. Posteriormente, realizou-se a pasteurização do creme a temperatura de $85^{\circ} \mathrm{C}$ por 5 minutos, logo este foi resfriado a $10{ }^{\circ} \mathrm{C}$ para adição da cultura láctea. $\mathrm{O}$ processo de fermentação do creme de leite ocorreu com a utilização dos microrganismos Lactococcus lactis ssp. lactis e Lactococcus lactis ssp. cremoris (DELVO ${ }^{\circledR}$ - TEC LL 50A DSL, DSM, Holanda) na proporção de $1 \%$ a $3 \%$, e foi armazenado a $15{ }^{\circ} \mathrm{C}$ a $20^{\circ} \mathrm{C}$ durante 18 horas.

No dressing foi adicionado a substância prebiótica inulina (Orafti ${ }^{\circledR} \mathrm{HP}$, Beneo, Alemanha) na proporção de $3 \%$ a $5,5 \%$ com 
acréscimo de leite desnatado $(100 \mathrm{~mL} / \mathrm{kg}$ de produto final) para efetuar a diluição deste ingrediente. A porcentagem (1,15\% a 1,60\%) de sal light NEVE (Guss Industrial, Brasil) foi adicionada, $\mathrm{NaCl}$ (1\% a 1,9\%) (Cisne, Brasil) e sorbato de potássio $(0,15 \%$ a $0,85 \%)$ (All Chemistry - São Lucas), conforme indicado na Tabela 1. O dressing foi pasteurizado novamente a $85^{\circ} \mathrm{C}$ por 5 minutos e resfriado a $5 \pm 2{ }^{\circ} \mathrm{C}$ por 12 horas. Após este processo, foi adicionado de $7 \%$ a $9 \%$ de dressing na massa seguiu-se o envase e a estocagem sob refrigeração por 42 dias a $5 \pm 2{ }^{\circ} \mathrm{C}$.

A Figura 1 apresenta o fluxograma com as etapas de processamento para elaboração do queijo tipo cottage.

\section{Caracterização físico-química e micro- biológica}

O queijo tipo cottage foi avaliado nos tempos $0,7,14,21,28,35$ e 42 dias de armazenamento de acordo com ensaios microbiológicos e físico-químico (acidez), para determinar a qualidade e a vida útil do produto. A acidez, foi avaliada apenas no queijo tipo cottage desenvolvido neste trabalho. Quanto aos ensaios microbiológicos foram determinados contagem total de Staphylococcus coagulase positiva (ISO 6888-1: 1999), coliformes termotolerantes e bolores e leveduras (BRASIL, 2003), detecção de Salmonella spp (método 2011.03) e Listeria monocytogenes (método 2004.02), de acordo com Horwitz (2005c, 2005d) respectivamente. Todas as análises foram realizadas em triplicata.

Quanto aos parâmetros físico-químicos, foram determinados $\mathrm{pH}$, acidez em ácido lático, lipídios, umidade, proteínas, resíduo mineral fixo, matéria gorda no extrato seco, para estes foram utilizados os métodos oficiais para as referidas análises, descritos na Instrução Normativa $n^{\circ}$ 68, de 12 de dezembro de 2006 (BRASIL, 2006). O ensaio de fibra foi determinando através da metodologia de fruto-oligossacarídeos (método 999.03) e o ensaio de sódio foi quantificado de acordo com o método 985.35 e 984.27 , ambos baseados na AOAC (2005) (HORWITZ, 2005a; HORWITZ, 2005b). A quantificação da lactose do produto final foi realizada

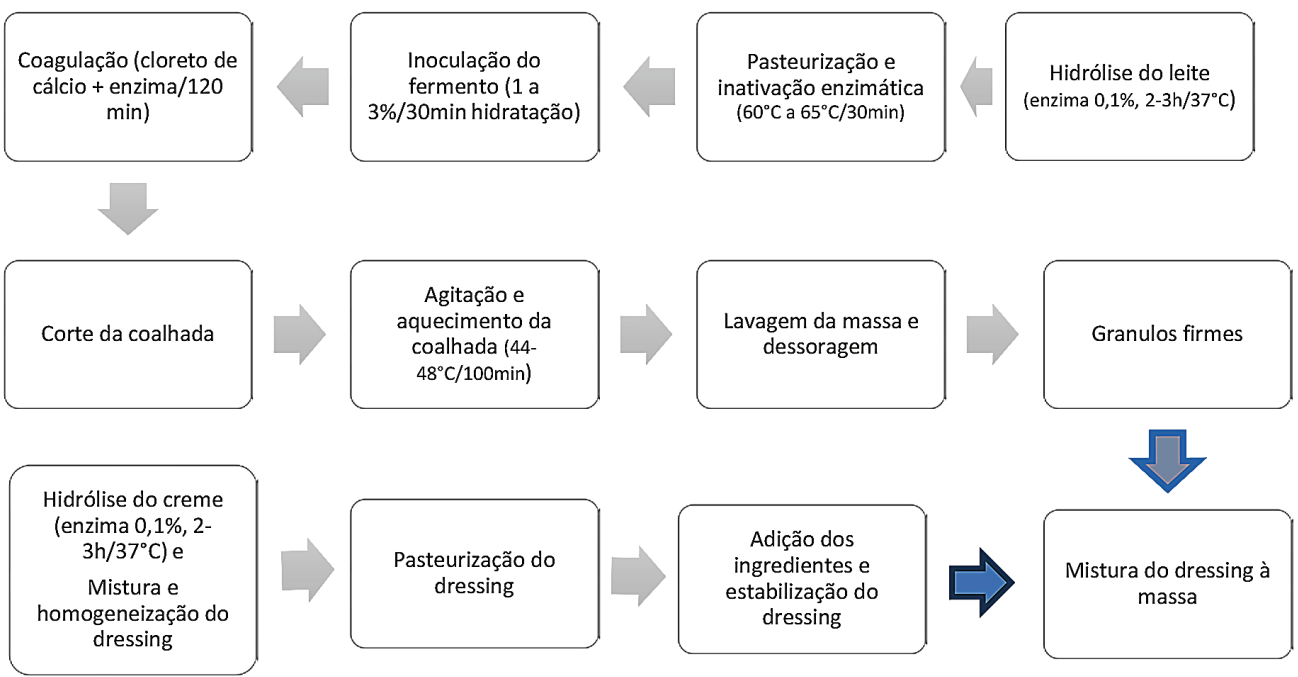

Figura 1 - Fluxograma do processamento na elaboração do queijo tipo cottage 
utilizando cromatografia líquida de alta eficiência (HPLC), de acordo com método adaptado de Harvey (1988). Todas as análises foram realizadas em triplicata.

\section{Análise sensorial}

O teste de aceitabilidade foi realizado com um grupo de julgadores não treinados $(\mathrm{n}=50)$, através de escala hedônica estruturada de nove pontos (1 - desgostei extremamente a 9 - gostei extremamente), para os atributos cor, odor, sabor, textura e aceitabilidade global. Já para a avaliação da intenção de compra foi utilizada uma escala estruturada de cinco pontos ( 1 - certamente não compraria a 5 - certamente compraria). Aproximadamente $25 \mathrm{~g}$ de cada amostra à $5 \pm 1{ }^{\circ} \mathrm{C}$, foram apresentadas monadicamente em copos plásticos $(50 \mathrm{~mL})$ codificados com números de três dígitos aleatórios (MINIM, 2010). Colheres plásticas e torradas sem sal foram oferecidas junto às amostras.

\section{Análise estatística}

Os resultados obtidos nas análises físico-químicas foram analisados através do software STATISTICA 7.0 (Statsoft, Tulsa, EUA). A análise de variância (ANOVA) foi aplicada para determinar as diferenças significativas $(p<0,05)$ entre as amostras, seguida pelo teste de Tukey.

\section{RESULTADOS E DISCUSSÃO}

\section{Caracterização físico-química e microbiológica}

A Tabela 2 apresenta a média dos valores encontrados para teor de umidade, lipídios, proteína, $\mathrm{pH}$, acidez, fibra alimentar, matéria gorda no extrato seco,sódio e lactose do queijo tipo cottage (com redução de sódio, gordura, lactose e adição de inulina) desenvolvido e o queijo controle durante a estocagem a $5 \pm 2{ }^{\circ} \mathrm{C}$.

Para os queijos avaliados, houve diferença $(\mathrm{p}<0,05)$ para os parâmetros de umidade, lipídios, sódio, proteína e acidez titulável. O queijo controle apresentou maiores médias para estas características, exceto para proteína e umidade. Os diferentes teores de umidade e lipídios avaliados podem ser explicados pela adição da substância prebiótica inulina ao dressing do queijo. A inulina

Tabela 2 - Composição físico-química do queijo tipo cottage (com redução de sódio, gordura, lactose e adição de inulina) e queijo controle

\begin{tabular}{lcc}
\hline \multicolumn{1}{c}{ Parâmetros analisados } & Queijo tipo cottage & Queijo controle \\
\hline Umidade (\%) & $79,22^{\mathrm{a}}$ & $74,33^{\mathrm{b}}$ \\
Proteína (\%) & $13,52^{\mathrm{a}}$ & $11,13^{\mathrm{b}}$ \\
Lipídios (\%) & $2,75^{\mathrm{a}}$ & $3,95^{\mathrm{b}}$ \\
Fibra Alimentar (\%) & 5,31 & - \\
Matéria gorda no extrato seco (\%) & $19,33^{\mathrm{a}}$ & $19,00^{\mathrm{a}}$ \\
Sódio (mg/100g) & $219,5^{\mathrm{a}}$ & $372^{\mathrm{b}}$ \\
Acidez titulável (\% de ácido lático) & $0,05^{\mathrm{a}}$ & $0,25^{\mathrm{b}}$ \\
pH & $4,61^{\mathrm{a}}$ & $4,82^{\mathrm{a}}$ \\
Lactose (g/100g) & $<0,1^{\mathrm{a}}$ & $<0,1^{\mathrm{a}}$ \\
\hline
\end{tabular}

Os valores apresentados são as médias dos parâmetros avaliados. Na mesma linha, letras minúsculas sobrescritas indicam as diferenças $(\mathrm{p}<0,05)$ entre os queijos. 
além de função prebiótica, também foi utilizada como substituto parcial de gordura, reduzindo $30 \%$ quando relacionada com o queijo controle.

Inulina e oligofrutoses possuem baixo valor calórico em função de ligações $\beta$ (1-2) entre as moléculas de frutose. As ligações tornam estas substâncias não digeríveis por enzimas intestinais humanas. Assim, estes prebióticos passam pelo trato gastro digestório sem serem metabolizados. Estudos indicam que a inulina e oligofrutoses são completamente fermentados no cólon e produzem ácidos graxos de cadeia curta e lactatos que são metabolizados e contribuem com o valor calórico de 1,5 kcal/g (NINESS, 1999).

Constituída de cadeias longas, a inulina é menos solúvel que a oligofrutose e, quando dispersa na água ou no leite, forma micro cristais que interagem para dar origem a uma textura cremosa. Consequentemente é empregada como substituto de gordura em produtos lácteos, patês, molhos, recheios, coberturas, sobremesas congeladas e produtos de panificação (NINESS, 1999). Além disso, no dressing do queijo houve maior acréscimo de leite desnatado $(100 \mathrm{~mL})$ para efetuar a diluição do ingrediente prebiótico. Isto explica a razão do queijo tipo cottage (com redução de sódio, gordura, lactose e adição de inulina) ter apresentado maior teor de umidade em relação ao queijo controle.

A coagulação ácida necessária para produzir o queijo tipo cottage consegue-se através do processo de fermentação com a adição regular de culturas mesofilícas Lactococcus lactis subsp. cremoris e Lactococcus lactis subsp. lactis. Uma das vantagens da coagulação ácida ocorre, pois a produção do coágulo menor e leve, se comparado ao queijo produzido por coagulação enzimática, facilita a digestibilidade das proteínas (OBANDO et al., 2010). A baixa acidez após a fabricação obtida neste estudo para ambos os queijos ocorreu em virtude das etapas processo de fabricação do queijo tipo cottage. Durante a produção, a massa foi aquecida até $50{ }^{\circ} \mathrm{C}$, o que ocasionou a inativação da cultura mesofílica starter adicionada. Além disso, no final do processo a massa passou por três lavagens consecutivas, contribuindo também para redução da acidez.

O conteúdo de fibras no produto foi outro diferencial, pois os queijos tipo cottage convencionais não apresentam fibras em sua composição e o deste estudo contém em torno de $5,4 \%$. O produto pode ser considerado como "fonte de fibras", pois apresenta mais que $2,5 \mathrm{~g}$ de fibra por porção do alimento pronto para o consumo, conforme a RDC $n^{\circ} 54$ de 2012 (ANVISA, 2012). A inulina pertence à classe de carboidratos denominados frutanos e são considerados ingredientes funcionais, uma vez que exercem influência sobre processos fisiológicos e bioquímicos no organismo, resultando em melhoria da saúde e em redução no risco de aparecimento de diversas doenças (SAAD, 2006). Niness (1999) destaca que a inulina tem sido amplamente utilizada nas indústrias de alimentos para substituírem gorduras e açúcares, reduzindo assim o teor calórico.

De acordo com a Tabela 2, observa-se a redução de $41 \%$ de sódio e $30 \%$ de gordura no queijo desenvolvido quando comparado com o controle. Segundo a RDC n 54 de 2012 (ANVISA, 2012), quando há uma redução mínima de $25 \%$ destes atributos, podese denominar o produto como light.

Ao avaliar o teor de lactose nos produtos, observa-se que não houve diferença significativa entre as amostras. Por isso, pressupõe-se que no queijo com uso de fermento na coalhada e na maturação do dressing não há necessidade de utilizar a enzima lactase. Na Tabela 3 observou-se a variação da acidez entre 0,04 e $0,16 \mathrm{~g} / 100 \mathrm{~g}$ determinada em ácido lático ao longo dos 42 dias de armazenamento do produto. A acidez do queijo tende a aumentar com a multiplicação dos microrganismos no decorrer do período de armazenamento, beneficiando o 
produto por inibir a microbiota patogênica (WOLFSCHOON- POMBO; LIMA, 1989).

Tabela 3 - Variação da acidez em ácido lático em 42 dias de armazenamento

\begin{tabular}{cc}
\hline $\begin{array}{c}\text { Tempo após } \\
\text { fabricação (dias) }\end{array}$ & $\begin{array}{c}\text { Acidez (ácido lático) } \\
\mathrm{g} / 100 \mathrm{~g}\end{array}$ \\
\hline 0 & $0,04 \pm 0,02$ \\
7 & $0,06 \pm 0,13$ \\
14 & $0,06 \pm 0,01$ \\
21 & $0,05 \pm 0,05$ \\
28 & $0,10 \pm 0,63$ \\
35 & $0,10 \pm 0,89$ \\
42 & $0,16 \pm 0,52$ \\
\hline
\end{tabular}

Conforme VIANA (2002), a acidez abaixo de $0,3 \mathrm{~g} / 100 \mathrm{~g}$ ao longo da estocagem é considerada boa característica por conferir ao produto um sabor mais suave e maior durabilidade.

Na Tabela 4 estão apresentados os resultados microbiológicos do queijo tipo cottage desenvolvido.

Os resultados obtidos na contagem de coliformes termotolerantes variou de $<10$ a $1,0 \times 10^{2} \mathrm{UFC} / \mathrm{g}$, estando de acordo com a Resolução - RDC nº 12/2001 (ANVISA,
2001), que determina um limite máximo de $5,0 \times 10^{3} \mathrm{UFC} / \mathrm{g}$ para estes microrganismos de queijo de muita alta umidade com bactérias láticas viáveis. As bactérias do grupo coliforme servem como indicadores de contaminação e a presença desses microrganismos em alimentos processados indica falhas de higienização no processamento, utensílios, equipamentos ou manipuladores e/ou contaminação pós processamento.

Conforme a Tabela 4, os microrganismos Staphylococcus coagulase positiva, Listeria monocytogenes e Salmonella spp., apresentaram-se coerentes com a legislação vigente RDC n ${ }^{\circ} 12 / 2001$, que preza o limite máximo de $10^{2} \mathrm{UFC} / g$ para Staphylococcus coagulase positiva e ausência em 25 g para Listeria e Salmonella (ANVISA, 2001).

$\mathrm{O}$ número de bolores e leveduras variou entre $1,0 \times 10^{2}$ a $4,4 \times 10^{3} \mathrm{UFC} / \mathrm{g}$, adequando-se a legislação vigente. Embora a RDC $n^{\text {o }}$ 12/2001 (ANVISA, 2001) não apresente limites para bolores e leveduras para nenhum alimento, a Portaria no 146/1996 do MAPA, onde contém os Regulamentos Técnicos de Identidade e Qualidade dos Produtos Lácteos, determina o limite estabelecido para queijos de umidade superior a $55 \%$ de $5,0 \times 10^{3}$ UFC/g. Assim sendo, o produto desenvolvido neste estudo está apto para ser consumido

Tabela 4 - Parâmetros microbiológicos para formulação de queijo cottage

\begin{tabular}{c|c|c|c|c|c}
\hline $\begin{array}{c}\text { Tempo } \\
\text { após } \\
\text { fabricação } \\
\text { (dias) }\end{array}$ & $\begin{array}{c}\text { Coliformes/g } \\
\left(45^{\circ} \mathrm{C}\right) \\
(\mathrm{UFC} / \mathrm{g})\end{array}$ & $\begin{array}{c}\text { Staphylococcus } \\
\text { coagulase } \\
\text { positiva } \\
(\mathrm{UFC} / \mathrm{g})\end{array}$ & $\begin{array}{c}\text { Listeria } \\
\text { monocytogenes } \\
\text { Ausência } \\
\mathrm{em} 25 \mathrm{~g}\end{array}$ & $\begin{array}{c}\text { Salmonella } \mathrm{ssp} . \\
/ 25 \mathrm{~g}\end{array}$ & $\begin{array}{c}\text { Bolores } \\
\mathrm{e} \\
\text { leveduras } \\
(\mathrm{UFC} / \mathrm{g})\end{array}$ \\
\hline 0 & $<10$ & $<1,0 \times 10^{2}$ & Ausência & Ausência & $1,2 \times 10^{2}$ \\
7 & $<10$ & $<1,0 \times 10^{2}$ & Ausência & Ausência & $>1,5 \times 10^{2}$ \\
14 & $<40$ & $<1,0 \times 10^{2}$ & Ausência & Ausência & $1,0 \times 10^{2}$ \\
21 & $<10$ & $<1,0 \times 10^{2}$ & Ausência & Ausência & $1,2 \times 10^{3}$ \\
28 & $<10$ & $<1,0 \times 10^{2}$ & Ausência & Ausência & $6,3 \times 10^{2}$ \\
35 & $<10$ & $<1,0 \times 10^{2}$ & Ausência & Ausência & $9,3 \times 10^{2}$ \\
42 & $1,0 \times 10^{2}$ & $<1,0 \times 10^{2}$ & Ausência & Ausência & $4,4 \times 10^{3}$ \\
\hline
\end{tabular}


e indica as boas condições de processamento e/ou armazenamento.

$\mathrm{O}$ crescimento de fungos nos queijos pode reduzir a quantidade de ácido lático, favorecendo o desenvolvimento de outros microrganismos potencialmente patógenos e ocasionando alterações nas características sensoriais (SALVADOR, 2001). De modo geral, os bolores e leveduras produzem enzimas que hidrolisam as proteínas, lipídios e carboidratos, dando origem a degradações que promovem modificações na coloração, aparência desagradável, perda de sabor, podendo, em alguns casos, produzir metabólitos tóxicos conhecidos como micotoxinas, o que pode torná-los impróprios para o consumo (LOURENÇO; SOUSA, 2005).

Portanto o queijo tipo cottage com adição de fibras e redução de sódio e gordura, apresentou-se estável em relação aos parâmetros microbiológicos e físico-químicos durante 42 dias de armazenamento.

\section{Avaliação sensorial}

Dos 50 provadores não treinados, 42 responderam que gostam de queijo tipo cottage e 8 que não gostam. Por isso, nesta pesquisa consideraram-se apenas os julgadores que apreciam o produto, ou seja, 42 pessoas. Destaca-se que esta avaliação foi realizada apenas com o queijo tipo cottage com redução, desenvolvido neste trabalho.

Ao perguntar aos julgadores quanto à frequência que consomem este tipo de queijo, 1 respondeu diariamente, 7 semanalmente, 6 mensalmente, 22 pessoas esporadicamente e 14 não consomem. Quando perguntados quanto ao interesse de adquirir o produto se estivesse à venda, 45,2\% responderam que "certamente comprariam". Quanto ao hábito de consumir alimentos funcionais, $85,7 \%$ dos provadores responderam que possuem o hábito.

A Figura 2 apresenta os resultados obtidos, sendo que $4,8 \%$ optou pelo termo "gostei extremamente", 57,1\% pelo termo "gostei muito" e 38,1\% pelo termo "gostei moderadamente", o que demonstra que o alimento foi bem aceito sensorialmente.

As notas obtidas utilizando a escala hedônica (Figura 2) apresentaram resultados

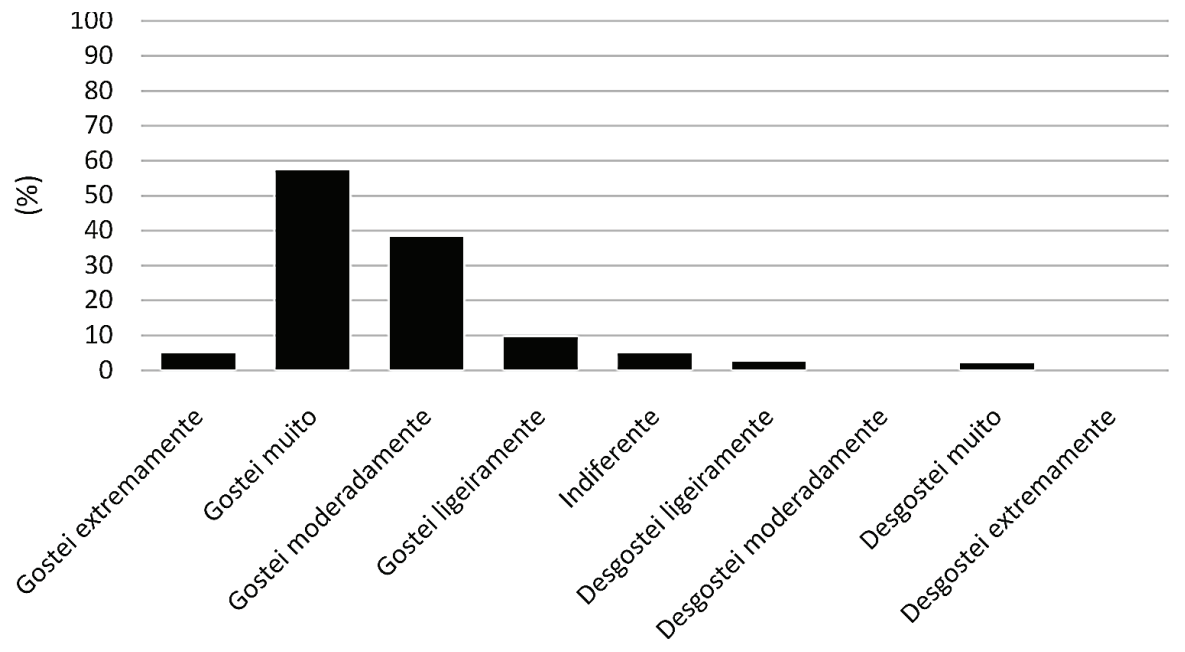

Figura 2 - Resultados do teste de aceitação em escala hedônica 
bastante satisfatórios para o produto (maior parte dos provadores optaram pelos escores "gostei muito" e "gostei moderadamente"), avaliando que a adição complementar da inulina, redução de sódio e gordura e a hidrólise da lactose não interferiram nos resultados.

\section{CONCLUSÕES}

O queijo cottage apresentou quantidade de fibras superior a $2,5 \%$, podendo ser considerado como "fonte de fibras". Quanto à redução de sódio e gordura, o produto pode ser considerado um produto light. Em relação às análises microbiológicas e de acidez, o produto apresentou estabilidade durante os 42 dias verificados.

O queijo cottage com redução de sódio, gordura, lactose e adição de inulina teve uma boa aceitação pelos provadores. A adição complementar de fibras não afetou negativamente a sensorialidade, resultando em um produto com boas perspectivas para comercialização.

\section{REFERÊNCIAS}

ANTUNES, A. E. C.; PACHECO, M. T. B. Leite para adultos: Mitos e fatos frentes à ciência. São Paulo: Varella, 2009. 457p.

AGÊNCIA NACIONAL DE VIGILÂNCIA SANITÁRIA (ANVISA). Resolução de Diretoria Colegiada, RDC n ${ }^{\circ} 12$, de 02 de Janeiro de 2001. Aprova o regulamento sobre padrões microbiológicos para alimentos. Diário Oficial da República Federativa do Brasil, n. 7, 10 jan. 2001. Seção 1, p. 45-53.

AGÊNCIA NACIONAL DE VIGILÂNCIA SANITÁRIA (ANVISA). Resolução de Diretoria Colegiada, RDC $\mathrm{n}^{\circ} 54$, de 12 de novembro de 2012. Aprova o regulamento técnico sobre informação nutricional complementar. Diário Oficial da República Federativa do Brasil, $n^{\circ}$ 219, 13 nov. 2012. Seção 1, p. 45-53.
ASSOCIATION OF OFFICIAL ANALYTICAL CHEMISTS (AOAC). Official methods of analysis of AOAC International. 18. ed. Gaithersburg: AOAC, 2005

BRASIL. Ministério da Agricultura, Pecuária e Abastecimento. Portaria ${ }^{\circ}$ 146, de 07 de março de 1996. Aprova os Regulamentos Técnicos de Identidade e Qualidade de Queijos. Diário Oficial da República Federativa do Brasil, 11 mar. 1996. Seção 1, p. 3977.

BRASIL. Ministério da Agricultura, Pecuária e Abastecimento. Departamento de Inspeção de Produtos de Origem Animal. Instrução Normativa $n^{0} 62$, de 26 de agosto de 2003. Oficializa os métodos analíticos oficiais para análises microbiológicas para controle de produtos de origem animal e água. Diário Oficial da República Federativa do Brasil, Brasília, 18 set. 2003. Seção 1, p. 14.

BRASIL. Ministério da Agricultura, Pecuária e Abastecimento. Instrução Normativa $\mathrm{n}^{\mathrm{o}}$ 68, de 12 de dezembro de 2006. Oficializa os métodos analíticos oficiais físico-químicos, para controle de leite e produtos lácteos. Diário Oficial da República Federativa do Brasil, Brasília, 14 dez. 2006. Seção 1, p. 8.

BRITO, C. et al. Queso cottage elaborado con cultivo láctico redi-set y dvs, usando crema lactea homogeneizada y sin homogeneizar. Revista Chilena de Nutrición, v. 33, n. 1, Santiago, 2006.

HARVEY, J. A high performance liquid chromatography method for lactose determination in milk. Australian Journal of Dairy Technology, v. 43, p. 19-20, 1988.

HORWITZ, W. (Ed.). Official methods of analysis of the Association of Official Analytical Chemists. 18th ed. Gaithersburg, Maryland: AOAC, 2005a. Cap. 45, met. 45.4.06B, p. 92-94. Meagazyme-Fructan HK Procedure for the measurement os Fructo- 
oligosacharides and Fructan Polysacaharide A modification of AOAC Method 999.03.

HORWITZ, W. (Ed.). Official methods of analysis of the Association of Official Analytical Chemists. 18 th ed. Gaithersburg, Maryland: AOAC, 2005b. Chapter 50, methods. 985.35 e 984.27 , p. 15-18.

HORWITZ, W. (Ed.). Official methods of analysis of Association of Oficial Analytical Chemists. 18th ed. Gaithersburg: AOAC, 2005c. AOAC Official Method 2011.03 - Metodologia Alternativa - Detection of Salmonella by the VIDASÂ ${ }^{\circledR}$ Easy Salmonella Assay (Easy SLM) with Chrom IDTM Salmonella (SM2) Agar in a Variety of Foods.

HORWITZ, W. (Ed.). Official methods of analysis of Association of Oficial Analytical Chemists. 18th ed. Gaithersburg: AOAC, 2005d. Official Method 2004.02 Listeria monocytogenes II (LMO2). AOAC Internacional. Cap. 17, p. 233-234.

ISO. International Standard Organization. 6888-1:1999. Microbiology of food and animal feeding stuffs - Horizontal method for the enumeration of coagulase positive staphylococci (Staphylococcus aureus and other species) Part 1: Technique using Baird-Parker agar medium. ISO 6888 - 1: 2009/Amd1:2003.

LOURENÇO, L. F. H.; SOUSA, C. L. Análise microbiológica e teste de aceitação de requeijão marajoara elaborado com leite de búfala. Higiene Alimentar, v. 19, n. 132, p. 84-88, junho 2005.

MANNING, T. S. et al. Microbial-gut interactions in health and disease. Prebiotics. Best Practice Research Clinical Gastroenterology, v. 18, n. 2, p. 287-298, 2004.

MINIM, V. P. R. Análise sensorial, estudos com consumidores. $2^{\mathrm{a}}$ ed. Universidade Federal de Viçosa. 2010.
NINESS, K. R. Inulin and oligofrutctose: What are they? American Society for Nutritional Sciences, n. 129, 1402S-6S.

OBANDO C. M. et al. Viabilidad de los microrganismos probióticos Lactobacillus casei 01, Lactobacillus acidophilus La-5, Bifidobacterium BB12 durante el almacenamiento de queso cottage. Vitae, v. 17, n. 2, 2010.

PARODIA, C. G. Desenvolvimento de queijo Cottage simbiótico. 2010. 120 p. Dissertação (mestrado em Ciência e Tecnologia dos Alimentos) - Universidade Federal de Santa Maria, Santa Maria, 2010.

RIBEIRO, E. P. et al. Desenvolvimento de queijo Minas frescal adicionado de Lactobacillus acidophilus produzido a partir de retentados de ultrafiltração. Ciência e Tecnologia de Alimentos, Campinas, v. 29, n. 1, p. 19-23,2009.

RODRIGUES, F. C. Lácteos especiais. Juiz de Fora: Concorde Editora Gráfica, 1999. 151 p.

SAAD, S. M. I. Probióticos e prebióticos: o estado da arte. Brazilian Journal of Pharmaceutical Sciences, v. 42, n. 1, 2006.

SALVADOR, M. et al. Avaliação da qualidade microbiológica de queijo prato e parmesão ralado. Boletim do Centro de Pesquisa e Processamento de Alimentos, Curitiba, v. 19, n. 1,2001

VIANA, G. A. et al. Produção de queijo tipo Cottage por acidificação direta do leite com ácido lático como coadjuvante no processo de fermentação. In: XIX Congresso Nacional de Laticínios, 57, 2002. Anais... Juiz de Fora: EPAMIG ILCT, 2002. p. 1-7.

WOLFSCHOON-POMBO, A. L.; LIMA, A. Extensão e profundidade da proteólise em queijo Minas frescal. Revista do Instituto de Laticínios Cândido Tostes, v. 44, n. 261-266, p. 50-52, 1989. 